\title{
Treat COVID-19, but Not Only COVID-19: Stroke Matters as Well
}

\author{
Petra Sedova a, b, c, d, e Robert D. Brown Jr. ${ }^{a}$ Tomas Bryndziar ${ }^{b, d, ~ e ~ J i r i ~ J a r k o v s k y ~}{ }^{f, g}$ \\ Ales Tomek $^{\mathrm{h}}$ Martin Sramek ${ }^{\mathrm{h}}$ Ondrej Skodai,j Tereza Sramkova ${ }^{\mathrm{h}}$ \\ Simona Littnerovaf,g Robert Mikulik ${ }^{b, d, e}$ \\ aDepartment of Neurology, Mayo Clinic, Rochester, MN, USA; 'b International Clinical Research Center, St. Anne's \\ University Hospital, Brno, Czech Republic; 'Department of Internal Medicine, Hematology and Oncology, University \\ Hospital Brno, Brno, Czech Republic; 'Department of Neurology, St. Anne's University Hospital, Brno, Czech Republic; \\ eFaculty of Medicine, Masaryk University, Brno, Czech Republic; fInstitute of Health Information and Statistics of \\ the Czech Republic, Prague, Czech Republic; IInstitute of Biostatistics and Analyses, Faculty of Medicine, Masaryk \\ University, Brno, Czech Republic; hDepartment of Neurology, Charles University in Prague, 2nd Faculty of Medicine and \\ Motol University Hospital, Prague, Czech Republic; 'Neurological Department and Stroke Unit, Jihlava Hospital, Jihlava, \\ Czech Republic; 'Department of Neurology, University Hospital Kralovske Vinohrady and Charles University in Prague, \\ Prague, Czech Republic
}

\section{Keywords}

COVID-19 · Czech Republic · Intravenous thrombolysis ·

Mechanical thrombectomy $\cdot$ Stroke

\begin{abstract}
Introduction: During the COVID-19 pandemic, studies reported less number of hospitalizations for acute stroke and reduction in the use of recanalization treatments. This study analyzes nationwide data on stroke admissions and management in the Czech Republic during the first wave of the COVID-19 pandemic. Methods: We compared the early COVID-19 pandemic (March-May 2020) with the pre-pandemic period (January-February 2020 and March-May 2019): (a) the National Register of Reimbursed Health Services provided volume of all admissions for subarachnoid hemorrhage
\end{abstract}

karger@karger.com www.karger.com/ced

Karger $\stackrel{\text { ' }}{5}$

GOPEN ACCESS
(C) 2021 The Author(s)

Published by S. Karger AG, Basel

This is an Open Access article licensed under the Creative Commons Attribution-NonCommercial-4.0 International License (CC BY-NC) (http://www.karger.com/Services/OpenAccessLicense), applicable to the online version of the article only. Usage and distribution for commercial purposes requires written permission.
(SAH), intracerebral hemorrhage (ICH), and ischemic stroke (IS), and volume of recanalization treatments (intravenous thrombolysis [IVT] and mechanical thrombectomy [MT]); (b) Registry of Stroke Care Quality provided door-to-needle time (DNT), onset-to-door time (ODT), and stroke severity at admission (National Institutes of Health Stroke Scale, NIHSS) for IS. Results: During the pandemic (March-May 2020), the peak number of COVID-19 patients treated in Czech hospitals was 39 per million. In March-May 2020 versus MarchMay 2019, hospital admissions decreased as follows: stroke overall by $14 \%(p<0.001)$, IS by $14 \%(p<0.001)$, SAH by $15 \%$ $(p=0.07)$, and $\mathrm{ICH}$ by $7 \%(p=0.17)$. The mean age was 74 years versus 74 years $(p=0.33)$, and $52 \%$ versus $51 \%$ were men $(p=0.34)$. The volumes of IVT and MT decreased by $14 \%$ $(p=0.001)$ and $19 \%(p=0.01)$, respectively. The proportions of all IS patients receiving IVT or MT remained unchanged, 
with, respectively, $17 \%$ versus $17 \%$ receiving IVT ( $p=0.86$ ) and $5 \%$ versus $5 \%$ receiving MT ( $p=0.48)$. DNT and ODT were 24 versus $25 \min (p=0.58)$ and 168 versus $156 \min (p=0.23)$, respectively. NIHSS at admission did not differ (6 vs. $6 ; p=$ 0.54). Conclusion: Even with a low burden of COVID-19 during the first wave and no change in organization and logistics of stroke services, stroke admissions and volume of recanalization treatments decreased. Public health communication campaigns should encourage people to seek emergency medical care for stroke symptoms during the COVID-19 pandemic.

(C) 2021 The Author(s)

Published by S. Karger AG, Basel

\section{Introduction}

Several recent publications reported a decrease in the number of hospital admissions for acute cardiovascular diseases (ischemic stroke [IS], acute coronary syndrome, and myocardial infarction) during the COVID-19 pandemic [1-7]. Worsening of stroke management measures has also been reported, such as decreased volumes of mechanical thrombectomy (MT) and intravenous thrombolysis (IVT) and increased time to reperfusion therapy for IS $[5,8,9]$. The reasons for this are not entirely clear, but 2 main explanations have been proposed: (1) overwhelmed and thus unavailable health-care system, or (2) available but, because of patients' fear of infection, unused health-care system.

In response to the COVID-19 pandemic, the Czech Republic (CR) adopted a national state of emergency on March 12, 2020. This likely contributed to the exceptionally low initial burden of SARS-CoV-2 infection in the CR during the first wave but may have negatively affected the use of health services.

The aim of this study is to evaluate the hospital admissions for acute stroke and stroke management data in the CR using the complete nationwide data and to determine whether they were affected during the early stages of the COVID-19 pandemic (March-May 2020) when compared with the 2 immediately pre-pandemic months (January-February 2020) and the corresponding 3 months prior-year (March-May 2019).

\section{Materials and Methods}

We performed a nationwide retrospective study evaluating monthly volumes of patients hospitalized with a stroke diagnosis, IVT and MT volumes, based on an analysis of data from the $\mathrm{Na}$ - tional Register of Reimbursed Health Services (NRRHS) and the Registry of Stroke Care Quality (RES-Q) in the CR early in the COVID-19 pandemic.

Health-Care System and Stroke Care in the CR

The CR is a central European country with 10,708,982 inhabitants. The Czech health-care system is used by all citizens, and there is no personal expense incurred for health care received (universal health-care system). There is a network of 45 accredited stroke centers in the CR.

National Register of Reimbursed Health Services [10,11]

The NRRHS, part of the Institute of Health Information and Statistics (IHIS), collects and stores all data reported to health insurance companies by every health-care provider. All health insurance companies in the CR which reimburse health-care services from resources of the public health insurance system report all patient-related data to the NRRHS.

\section{Registry of Stroke Care Quality [12]}

Since 2018, data on all stroke patients discharged with a stroke diagnosis from all accredited stroke centers in the $\mathrm{CR}$ are collected in the international RES-Q [12] for 2 months every year (March or April, and October). Further, RES-Q includes stroke management data including time to initiation of reperfusion therapy (door-toneedle time [DNT] and onset-to-door time [ODT]), stroke severity (National Institutes of Health Stroke Scale [NIHSS]), and patient outcomes.

\section{Design}

Using the NRRHS, we analyzed data of all patients admitted to a hospital with a main diagnosis of stroke (diagnostic codes I60I64 according to the International Classification of Diseases, Tenth Revision [ICD-10]) in the CR in 2019 and 2020. The number of patients with subarachnoid hemorrhage (I60), intracerebral hemorrhage (I61), and acute IS (I63) was assessed; the admissions, proportion of each sex, and the mean age were calculated. The proportions of acute IS patients receiving IVT or MT were determined. The data were obtained and analyzed for the period between January 1, 2019 and May 31,2020. Using the RES-Q data, the time to initiation of reperfusion therapy (DNT and ODT) and stroke severity at admission (NIHSS) were evaluated for March 2019 and April 2020.

\section{Time Periods}

We compared the 3 initial pandemic months (March 1,2020May 31, 2020) with (1) the 2 months immediately preceding the pandemic (January 1, 2020-February 29, 2020) and (2) the corresponding 3 months in the previous year (March 1, 2019-May 31, 2019) to assess stroke care prior to COVID-19.

\section{Statistical Analysis}

Categorical variables are reported as absolute numbers and percentages. Continuous variables are presented as mean and standard deviation or median and interquartile range. The comparisons between years were performed using Fisher's exact test or the Mann-Whitney test, as appropriate. For overall volume analysis, the $p$ value is based on 2-sided Poisson means test. A $p$ value of $<0.05$ was considered statistically significant. All statistical analyses were produced using R Statistical Software (version 4.0.3). 
Fig. 1. Number of stroke patients admitted to hospitals in the CR in 2019 and 2020. CR, Czech Republic.

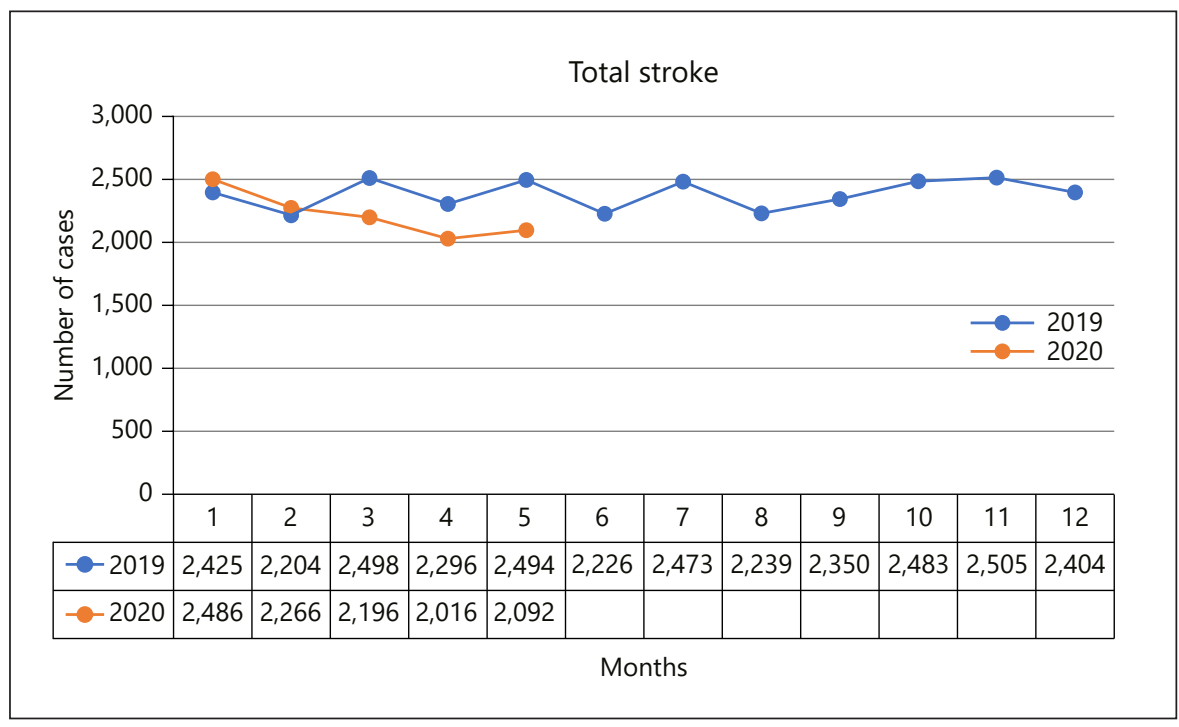

Table 1. Number of stroke patients admitted to hospitals in the CR in 2019 and 2020

\begin{tabular}{|c|c|c|c|c|c|}
\hline & $\begin{array}{l}1 \\
\text { March-May } 2019\end{array}$ & $\begin{array}{l}2 \\
\text { January-February } 2020\end{array}$ & $\begin{array}{l}3 \\
\text { March-May } 2020\end{array}$ & $\begin{array}{l}\text { Relative change in } \\
\text { monthly admissions } \\
3 \text { versus } 1 \\
3 \text { versus } 2\end{array}$ & $\begin{array}{l}p \text { value* } \\
3 \text { versus } 1 \\
3 \text { versus } 2\end{array}$ \\
\hline \multicolumn{6}{|l|}{ Stroke total } \\
\hline$N$ & 7,288 & 4,752 & 6,304 & $-14 \%$ & $<0.001$ \\
\hline Mean monthly admissions & 2,429 & 2,376 & 2,101 & $-12 \%$ & $<0.001$ \\
\hline Mean monthly admissions & 94 & 81 & 80 & $-1 \%$ & 0.96 \\
\hline \multicolumn{6}{|l|}{ ICH (I61) } \\
\hline$N$ & 863 & 554 & 806 & $-7 \%$ & 0.17 \\
\hline Mean monthly admissions & 287 & 277 & 269 & $-3 \%$ & 0.58 \\
\hline \multicolumn{6}{|l|}{ IS (I63) } \\
\hline$N$ & 6,142 & 4,037 & 5,258 & $-14 \%$ & $<0.001$ \\
\hline
\end{tabular}

CR, Czech Republic; SAH, subarachnoid hemorrhage; ICH, intracerebral hemorrhage; IS, ischemic stroke. * $p$ value based on 2 -sided Poisson means test.

\section{Results}

\section{Hospitalization}

By May 31, 2020, there were 9,268 total confirmed cases of SARS-CoV-2 infection (865 per million), the case fatality rate was low (320 total deaths, 30 deaths per million), and the peak number of COVID-19 patients treated in Czech hospitals was 39 per million (total capacity of all hospitals in the CR is 6,600 hospital beds per million) [13]. There were 7,$288 ; 4,752$; and 6,304 hospitalizations in the CR for any stroke (mean monthly admissions 2,429;
2,376; and 2,101), during the corresponding 3-month period in the pre-pandemic year (March-May 2019), the 2-month immediate pre-pandemic period (January-February 2020), and the 3-month pandemic period (MarchMay 2020), respectively. During the pandemic period (March-May 2020), stroke hospital admissions declined by $14 \%(p<0.001)$ and $12 \%(p<0.001)$ compared to the corresponding prior-year (March-May 2019) and the immediate pre-pandemic (January-February 2020) periods, respectively (Table 1). 
Fig. 2. a Number of IS patients admitted to hospitals in the CR in 2019 and 2020. b Number of SAH patients admitted to hospitals in the CR in 2019 and 2020. c Number of ICH patients admitted to hospitals in the CR in 2019 and 2020. CR, Czech Republic; $\mathrm{SAH}$, subarachnoid hemorrhage; $\mathrm{ICH}$, intracerebral hemorrhage; IS, ischemic stroke.

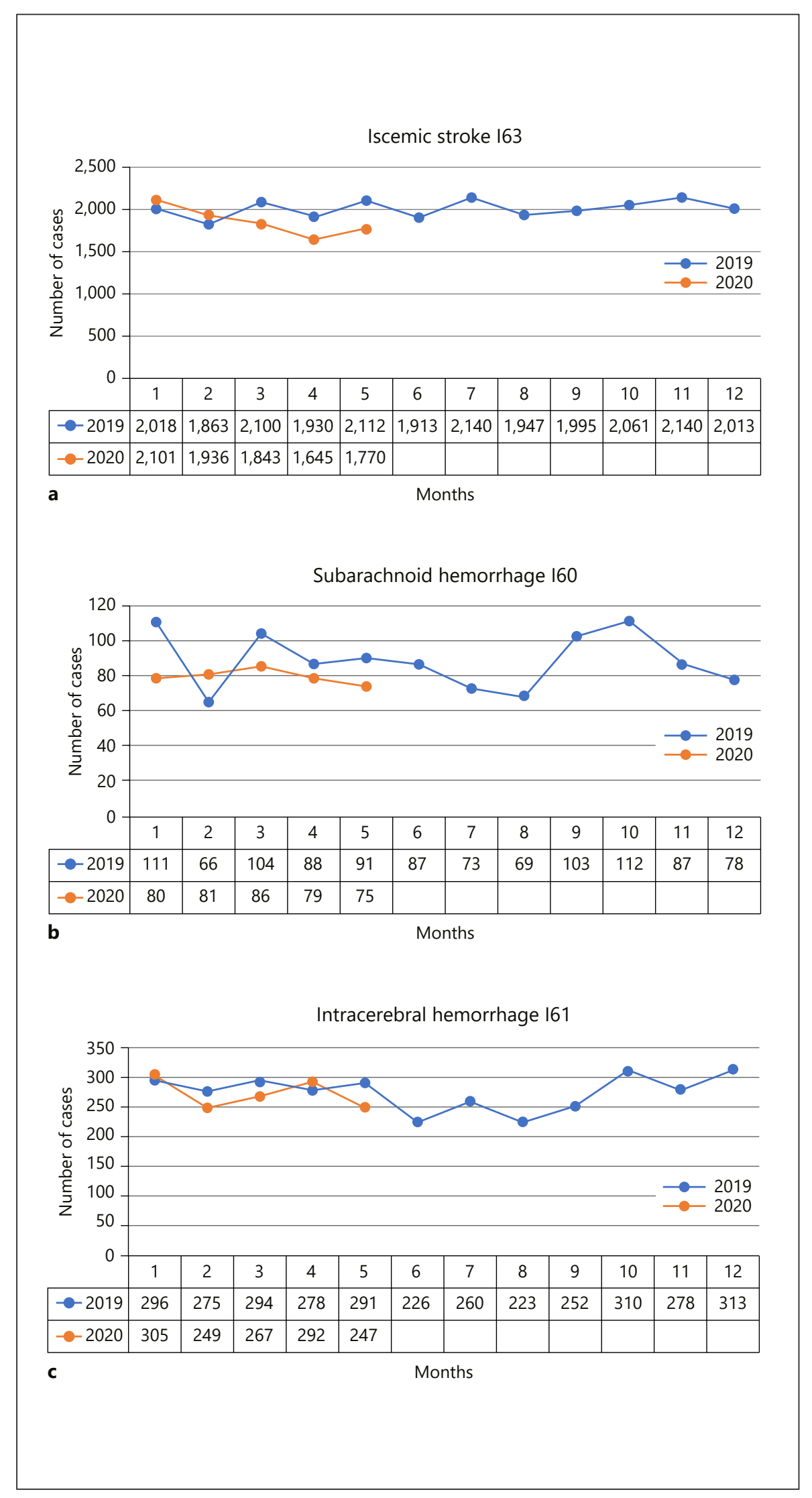


Table 2. Characteristics of IS patients (I63) and parameters of stroke care in 2019 and 2020 using NRRHS

\begin{tabular}{lllll}
\hline & $\begin{array}{l}1 \\
\text { March-May 2019 } \\
(N=6,142)\end{array}$ & $\begin{array}{l}2 \\
\text { January-February 2020 } \\
(N=4,037)\end{array}$ & $\begin{array}{l}3 \\
\text { March-May 2020 } \\
(N=5,258)\end{array}$ & $\begin{array}{l}p \text { value } \\
3 \text { versus 1 } \\
3 \text { versus 2 }\end{array}$ \\
\hline $\begin{array}{l}\text { Patient characteristics } \\
\text { Age, mean (SD) }\end{array}$ & $74(12)$ & $74(13)$ & $74(12)$ & 0.33 \\
Men, $n$ (\%) & $3,126(51)$ & $2,043(51)$ & $2,724(52)$ & 0.15 \\
Reperfusion therapy, $n(\%)$ & & & 0.34 \\
IVT & $1,056(17.2)$ & $702(17.4)$ & $911(17.3)$ & 0.26 \\
MT & $333(5.4)$ & $223(5.5)$ & $269(5.1)$ & 0.96 \\
& & & & $0.48^{\dagger}$ \\
\end{tabular}

IVT, intravenous thrombolysis; MT, mechanical thrombectomy; NRRHS, National Registry of Reimbursed Health Services; SD, standard deviation; IS, ischemic stroke. * $p$ value $0.86,0.96$ is referring to the comparison of percentages IVTs. $p$ value absolute count IVT: 3 versus 1: $p=0.001,3$ versus $2: p=0.004$. $p$ value is based on 2 -sided Poisson means test. ${ }^{\dagger} p$ value $0.48,0.40$ is referring to the comparison of percentages MTs. $p$ value absolute count MT: 3 versus 1: $p=0.010$, 3 versus 2: $p=0.017$. $p$ value is based on 2-sided Poisson means test.

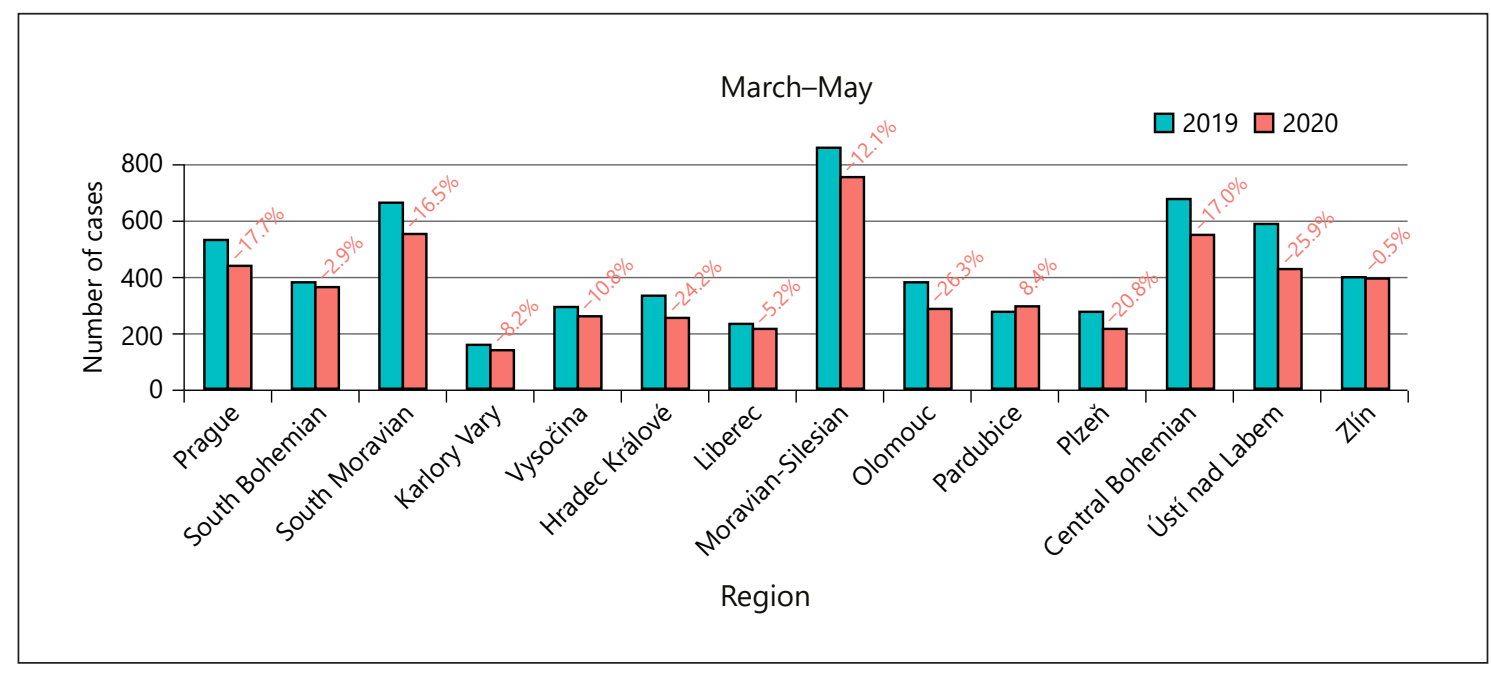

Fig. 3. Regional distributions - number of IS patients (I63) admitted to hospitals in 14 regions in the CR in MarchMay 2019 and March-May 2020. CR, Czech Republic; IS, ischemic stroke.

Monthly stroke hospitalizations are presented in Fig. 1, 2a-c, respectively. Figure 3 shows the decrease in IS admissions throughout the CR.

\section{IS Patient Characteristics and Stroke Management}

Patient characteristics are summarized in Table 2. During the early stages (March-May 2020) compared to 1 year earlier (March-May 2019), the IVT volumes declined by $14 \%(p=0.001)$, and the MT by $19 \%(p=0.01)$. The proportions of all IS patients receiving IVT or MT remained unchanged with, respectively, $17 \%$ versus $17 \%$ receiving IVT $(p=0.86)$, and $5 \%$ versus $5 \%$ receiving MT $(p=0.48)$.

Using the RES-Q (comparing April 2020 with March 2019 ), the time to initiation of reperfusion therapy remained unchanged (DNT [24 vs. $25 \mathrm{~min} ; p=0.58$ ] and ODT [168 vs. $156 \mathrm{~min} ; p=0.23]$ ). Stroke severity at admission also did not differ (NIHSS 6 vs. $6 ; p=0.54$ ) (Table 3). 
Table 3. IS (I63) patients and parameters of stroke care in 2019 and 2020 using RES-Q

\begin{tabular}{lccc}
\hline & $\begin{array}{c}\text { March 2019 } \\
(N=951)\end{array}$ & $\begin{array}{l}\text { April 2020 } \\
(N=1,033)\end{array}$ & p value \\
\hline $\begin{array}{l}\text { Time } \\
\text { DNT min., median, IQR }\end{array}$ & $25(15-35)$ & $24(16-33)$ & 0.58 \\
$\begin{array}{l}\text { ODT, min., median, IQR } \\
\text { Stroke severity } \\
\quad \text { NIHSS at admission; median, IQR }\end{array}$ & $156(84-430)$ & $168(85-474)$ & 0.23 \\
\hline
\end{tabular}

DNT, door-to-needle time; IQR, interquartile range; NIHSS, National Institutes of Health Stroke Scale; ODT, onset-to-door time; RES-Q, Registry of Stroke Care Quality; IS, ischemic stroke. The completeness of these variables in the RES-Q registry for March 2019 and April 2020 was as following: 90 and 91\% for NIHSS, 39 and 35\% for DNT, and $80 \%$ and $79 \%$ for ODT, respectively.

\section{Discussion}

We performed a nationwide study providing complete data on stroke in the CR during the first wave of the COVID-19 pandemic. The major findings were a significantly moderate reduction in acute stroke hospitalizations (14\%) and number of reperfusion therapies (IVT [14\%], MT [19\%]), but preservation of the frequency (proportions) and timing of advanced therapies for acute IS in the CR during the early stages of the COVID-19 pandemic (March-May 2020) as compared with 1 year earlier (March-May 2019). These results raise concern in light of low burden of COVID-19 cases in the CR in the early stages of the pandemic.

In the first wave compared with the previous year period, the decrease in hospitalization was observed in all stroke types, but only the decline in IS reached statistically significance $(14 \% ; p<0.001)$. The decline in subarachnoid hemorrhage $(15 \% ; p=0.07)$ was the same size but together with intracerebral hemorrhage $(7 \% ; p=0.17)$ was not statistically significant, which could be attributed to the lower number of hemorrhagic strokes and lack of power to detect a statistically significant change. The decrease in IS admissions was seen consistently throughout the CR (in 13 out of 14 regions). Our data are in line with other studies showing a reduction in acute IS admissions early in the COVID-19 pandemic in 2020 across stroke centers [6, 7] (China [5], the USA [14], Slovakia [15], Germany [16], Italy [3, 17], and Spain [18, 19]). Some centers reported a higher number of patients admitted with IS following local reorganization of stroke care (Italy) [6]. Interestingly, there was an increase in the number of large vessel occlusion ISs reported in New York [20]. The patients' characteristics (age, gender, and stroke severity) did not differ across the compared time periods. Stroke severity at admission (NIHSS) remained unchanged similar to other well-established European centers [6], which might indicate the resilience of acute stroke treatment. This is supported by a hypothesis that hospital overload related to high COVID-19 burden was not a key factor, since the decline was observed worldwide even in centers with low or non-existent COVID-19 patients [7]. Instead, this may have been predominantly the independent decision to not seek medical care due to fear of infection and lower social family interactions [16]. Otherwise, some studies report that patients with severe strokes sought medical care, but patients' fear of contracting coronavirus may have played a role in reduced hospitalizations for transient ischemic attacks and milder ISs $[4,9,16,21]$. This is of concern because even minor strokes carry an immediate high risk of recurrent stroke.

The nationwide data reported here suggest that there was no difference in the proportion of IS patients treated with reperfusion therapy, similar to other studies [15]. However, there was a decline in the absolute volume of reperfusion therapies (for both IVT and MT), as reported in other studies $[5,16,19]$. Our findings raise concerns that these patients may have missed acute therapy with proven efficacy in reducing death and stroke-related disability. Further, the median ODT and DNT were unchanged, which is contrary to the findings of other studies $[14,22]$ and suggests that once the health-care system was notified, all acute stroke treatment processes were unaffected.

The causes for decline in hospitalization for stroke during COVID-19 pandemic remain unexplained. It is suggested that both behavioral and infrastructural changes played a role [6]. The limitation of our study is that we cannot directly confirm how many cases did not activate emergency medical services while having stroke symp- 
toms. Therefore, we cannot completely rule out an alternative explanation for less stroke admissions, which could be lower stroke incidence, possibly due to reasons such as less physical stress due to self-isolation, less air pollution, traffic exposure, fast foods, lower exposure to infections (flu), and other viruses [7,23]. Other researchers oppose noting that it is unlikely that the incidence would decline and that an increase in stroke incidence could be anticipated, given the COVID-19 patients' predisposition for thromboembolic events $[24,25]$. Another limitation is that we could not ascertain whether the lower number of hospital visits was a result of people isolating at home during the pandemic having fewer events stroke events being witnessed by others who might have otherwise activated emergency medical services [7]. Local characteristics and local reorganization of stroke management might have also influenced local admission rates [6].

The main strength of our study is that it covers the entire CR. The study was conducted using the NRRHS, which by law records all reimbursed health services. The presented data about stroke hospitalization, age, gender, and reperfusion therapy (IVT, MT) are complete. We may have missed patients who died before hospitalization. The IHIS registries were previously validated $[10,11]$. There are known limitations concerning the 3 variables from RES-Q (DNT, ODT, and NIHSS). These data are available only for a proportion of patients, for those treated in accredited stroke centers (approximately 83\% of stroke patients) and only for 2 months every year. However, these limitations are restricted only to these 3 variables and do not alter the overall conclusions. Another strength is that in the CR there is a universal health care system free of charge to all patients. Therefore, even if there was change in economic status of residents due to the COVID-19 pandemic, this could not explain less hospital visits.

\section{Suggestions}

To avoid severe health as well as economic consequences from undiagnosed and untreated stroke, we suggest that

1. "stay at home" campaigns should be balanced by raising the public awareness of stroke symptoms and the importance of acute diagnosis and treatment (as well as for other time-sensitive diseases such as myocardial infarction);

2. patients with stroke symptoms should be encouraged to seek immediately medical care;

3. patients should be assured that hospitals take all preventive measures to prevent contracting coronavirus disease in the hospital settings.

\section{Conclusion}

During the first wave of the COVID-19 pandemic (March-May 2020), the CR had a low burden of SARSCoV-2 infections and the hospital capacity was not affected. However, a significant reduction in stroke hospitalizations and absolute volume of reperfusion therapies (IVT, MT) was reported. Importantly, the quality of stroke care as reflected by the proportion of acute IS patients receiving IVT or MT was unchanged. The "stay at home" campaigns should be balanced by raising public awareness of stroke symptoms and the importance of acute diagnosis and treatment to ensure that potentially effective stroke therapies continue despite the pandemic.

\section{Acknowledgment}

We thank Michaela Sedova, $\mathrm{PhD}$, and Iveta Selingerova, $\mathrm{PhD}$, for their help with the statistical analysis.

\section{Statement of Ethics}

The present study conforms to the guidelines issued in the Declaration of Helsinki. Informed consent requirement was waived by the Institutional Ethics Committees for this retrospective study using anonymized clinical data, with no direct patient contact. The Ethics Committees of St. Anne's University Hospital and Institute of Health Information and Statistics of the CR determined that this study does not constitute clinical research and is thus exempt from ethics committee review (communication received on January 13, 2021).

\section{Conflict of Interest Statement}

Tomas Bryndziar reports employment at Bristol-Myers Squibb outside the submitted work. All other authors have no conflict of interest to declare.

\section{Funding Sources}

Petra Sedova and Robert Mikulik were supported by the COST (European Cooperation in Science and Technology) Association, project No. CA18118, IRENE COST Action - Implementation Research Network in Stroke Care Quality and by the project No. LQ1605 from the National Program of Sustainability II and by the IRIS-TEPUS Project No. LTC20051 from the INTER-EXCELLENCE INTER-COST program of the Ministry of Education, Youth, and Sports of the Czech Republic.
Sedova et al. 


\section{Author Contributions}

P.S., R.D.B., and R.M. designed the study. S.L. and J.J. provided the data. P.S. and S.L. analyzed the data. P.S., R.D.B., R.M., and T.B. wrote the manuscript. All authors reviewed and critically revised this work.

\section{Data Availability Statement}

The data that support the findings of this study are available upon request from the corresponding author.

\section{References}

1 De Filippo O, D’Ascenzo F, Angelini F, Bocchino PP, Conrotto F, Saglietto A, et al. Reduced rate of hospital admissions for ACS during COVID-19 outbreak in Northern Italy. N Engl J Med. 2020 Jul 2;383(1):88-9.

2 Morelli N, Rota E, Terracciano C, Immovilli P, Spallazzi M, Colombi D, et al. The baffling case of ischemic stroke disappearance from the casualty department in the COVID-19 era. Eur Neurol. 2020;83(2):213-5.

3 Sacco S, Ricci S, Ornello R, Eusebi P, Petraglia $\mathrm{L}$, Toni $\mathrm{D}$, et al. Reduced admissions for cerebrovascular events during COVID-19 outbreak in Italy. Stroke. 2020 Dec;51(12):374650.

4 Solomon MD, McNulty EJ, Rana JS, Leong TK, Lee C, Sung SH, et al. The COVID-19 pandemic and the incidence of acute myocardial infarction. N Engl J Med. 2020 Aug 13; 383(7):691-3.

5 Zhao J, Li H, Kung D, Fisher M, Shen Y, Liu R. Impact of the COVID-19 epidemic on stroke care and potential solutions. Stroke. 2020 Jul;51(7):1996-2001.

6 Altersberger VL, Stolze LJ, Heldner MR, Henon H, Martinez-Majander N, Hametner C, et al. Maintenance of acute stroke care service during the COVID-19 pandemic lockdown. Stroke. 2021 May;52(5):1693-701.

7 Nogueira RG, Qureshi MM, Abdalkader M, Martins SO, Yamagami H, Qiu Z, et al. Global impact of COVID-19 on stroke care and intravenous thrombolysis. Neurology. 2021 Mar 25;96(23):e282438.

8 Kerleroux B, Fabacher T, Bricout N, Moïse M, Testud B, Vingadassalom S, et al. Mechanical thrombectomy for acute ischemic stroke amid the COVID-19 outbreak: decreased activity, and increased care delays. Stroke. 2020 Jul;51(7):2012-7.

9 Markus HS, Brainin M. COVID-19 and stroke-A global World Stroke Organization perspective. Int J Stroke. 2020 Jun;15(4):3614.
10 Sedova P, Brown RDJr, Zvolsky M, Kadlecova $\mathrm{P}$, Bryndziar T, Volny O, et al. Validation of stroke diagnosis in the national registry of hospitalized patients in the Czech Republic. J Stroke Cerebrovasc Dis. 2015 Sep;24(9): 2032-8.

11 Sedova P, Brown RD, Zvolsky M, Kadlecova P, Bryndziar T, Kubelka T, et al. Incidence of hospitalized stroke in the Czech Republic: the national registry of hospitalized patients. J Stroke Cerebrovasc Dis. 2017 May;26(5):97986.

12 Registry of Stroke Care Quality, RES-Q. 2016. [register online https://qualityregistry.eu]. FNUSA-ICRC. Accessed 2020 Jul 4.

13 Ritchie H, Ortiz-Ospina E, Beltekian D, Mathieu E, Hasell J, Macdonald B, et al. Coronavirus pandemic (COVID-19). Published online at OurWorldInData.org. Available from: https://ourworldindata.org/coronavirus. Accessed 2020 December 10.

14 Siegler JE, Heslin ME, Thau L, Smith A, Jovin TG. Falling stroke rates during COVID-19 pandemic at a comprehensive stroke center. J Stroke Cerebrovasc Dis. 2020 Aug;29(8): 104953.

15 Gdovinova Z, Vitkova M, Barakova A, Cvopova A. The impact of the COVID-19 outbreak on acute stroke care in Slovakia: data from across the country. Eur J Neurol. 2020 Nov 13.

16 Hoyer C, Ebert A, Huttner HB, Puetz V, Kallmünzer B, Barlinn K, et al. Acute stroke in times of the COVID-19 pandemic: a multicenter study. Stroke. 2020 Jul;51(7):2224-7.

17 Bersano A, Kraemer M, Touzé E, Weber R, Alamowitch S, Sibon I, et al. Stroke care during the COVID-19 pandemic: experience from three large European countries. Eur J Neurol. 2020 Sep;27(9):1794-800.

18 Rudilosso S, Laredo C, Vera V, Vargas M, Renú A, Llull L, et al. Acute stroke care is at risk in the era of COVID-19: experience at a comprehensive stroke center in Barcelona. Stroke. 2020 Jul;51(7):1991-5.
19 Tejada Meza H, Lambea Gil Á, Sancho Saldaña $A$, Martínez-Zabaleta $M$, Garmendia Lopetegui E, López-Cancio Martínez E, et al. Impact of COVID-19 outbreak in reperfusion therapies of acute ischaemic stroke in northwest Spain. Eur J Neurol. 2020 Dec;27(12): 2491-8.

20 Majidi S, Fifi JT, Ladner TR, Lara-Reyna J, Yaeger KA, Yim B, et al. Emergent Large vessel occlusion stroke during New York city;s COVID-19 outbreak: clinical characteristics and paraclinical findings. Stroke. 2020 Sep; 51(9):2656-63.

21 Diegoli H, Magalhães PSC, Martins SCO, Moro CHC, França PHC, Safanelli J, et al. Decrease in hospital admissions for transient ischemic attack, mild, and moderate stroke during the COVID-19 era. Stroke. 2020 Aug; 51(8):2315-21.

22 Siegler JE, Zha AM, Czap AL, Ortega-Gutierrez S, Farooqui M, Liebeskind DS, et al. Influence of the COVID-19 pandemic on treatment times for acute ischemic stroke: the Society of Vascular and Interventional Neurology Multicenter Collaboration. Stroke. 2020 Nov 30;52:407.

23 Katsouras C, Karapanayiotides T, Papafaklis M, Giannopoulos S, Ziakas A, Sianos G, et al. Greater decline of acute stroke admissions compared with acute coronary syndromes during COVID-19 outbreak in Greece: cere$\mathrm{bro} / \mathrm{cardiovascular}$ implications amidst a second wave surge. Eur J Neurol. 2020 Dec 8.

24 Helms J, Tacquard C, Severac F, Leonard-Lorant I, Ohana M, Delabranche X, et al. High risk of thrombosis in patients with severe SARS-CoV-2 infection: a multicenter prospective cohort study. Intensive Care Med. 2020 Jun;46(6):1089-98.

25 Mao L, Jin H, Wang M, Hu Y, Chen S, He Q, et al. Neurologic manifestations of hospitalized patients with coronavirus disease 2019 in Wuhan, China. JAMA Neurol. 2020 Jun 1; 77(6):683-90. 